\title{
Toxic effects of tamoxifen on the growth and respiratory activity of Bacillus stearothermophilus
}

\author{
C. Luxo ${ }^{\mathrm{a}, \mathrm{b}, *}$, A.S. Jurado ${ }^{\mathrm{b}}$, J.B.A. Custódio ${ }^{\mathrm{b}, \mathrm{c}}$, V.M.C. Madeira ${ }^{\mathrm{b}}$ \\ ${ }^{a}$ Laboratório de Microbiologia, Faculdade de Farmácia, Universidade de Coimbra, 3049-517 Coimbra, Portugal \\ ${ }^{\mathrm{b}}$ Centro de Neurociências, Universidade de Coimbra, 3049-517 Coimbra, Portugal \\ ${ }^{c}$ Laboratório de Bioquímica, Faculdade de Farmácia, Universidade de Coimbra, 3049-517 Coimbra, Portugal
}

\begin{abstract}
The anticancer drug tamoxifen (TAM) is used as first line therapy in breast cancer. Although tamoxifen is usually considered an estrogen antagonist, several studies suggest alternative mechanisms of action. Bacillus stearothermophilus has been used as a model to clarify the antiproliferative action of tamoxifen putatively related with drug-membrane interaction. According to previous data, TAM induces perturbation of membrane structure along with impairment of bacterial growth. The aim of this work was to correlate the effects of TAM on growth of intact B. stearothermophilus with the respiratory activity of isolated protoplasts of this bacteria. TAM inhibits bacterial growth and oxygen consumption of protoplasts as a function of concentration. Effects on oxygen consumption depend on the substrate used: NADH, allowing to study the full respiratory chain and ascorbate-TMPD to probe the final oxidase segment. The interaction of TAM with the respiratory components occurs at a level preceding the cytochrome oxidase segment. (C) 2001 Elsevier Science Ltd. All rights reserved.
\end{abstract}

Keywords: Tamoxifen; Bacillus stearothermophilus; Bacterial growth; Respiratory activity

\section{Introduction}

Tamoxifen (TAM) is a drug widely used in therapy and prevention of breast cancer (van den Koedjik et al., 1994). Although tamoxifen is usually considered an estrogen antagonist, there are multiple cellular effects not related with the oestrogen receptor (Kon, 1989). Owing to its lipophilic character, TAM is strongly incorporated into membranes, perturbing the physical behavior of native and model membranes (Custódio et al., 1993; Luxo et al., 1996) and changing membrane structural order of human breast cancer cells (Clarke et al., 1990). Presumably, these interactions play important roles, related either with the antitumoral activity or the cytotoxicity of this drug.

Microorganisms have often been used to clarify cellular mechanisms, namely membrane-mediated mechanisms.

Abbreviations: NADH, full respiratory system; TAM, tamoxifen; TMPD, $N, N, N^{\prime}, N^{\prime}$-tetramethyl- $p$-phenylenediamine.

* Corresponding author. Tel.: + 351-2398-52567; fax: +351-239852569 .

E-mail address: crisluxo@ci.uc.pt (C. Luxo).
The use of bacterial membranes to study structural interactions of lipophilic compounds has important advantages over eukaryotic membranes. Membrane organization of bacterial cells is comparatively simple; on the other hand, bacterial membranes are suitable models to study membrane-mediated toxic effects since a lipid bilayer is present, as in eukaryotic membranes, and bacterial lipids are structurally and functionally similar to the eukaryotic lipids.

TAM perturbs the physical behaviour of multilamellar liposomes prepared with polar lipids extracted from Bacillus stearothermophilus (Luxo et al., 1996). Moreover, the addition of TAM to the growth medium induces modifications of bacterial membrane lipid composition (Luxo et al., 1998) and characteristic membrane ultrastructural alterations (unpublished results) along with impairment of bacterial growth (Luxo et al., 1996).

In the present study, we seek to establish a correlation of TAM effects on growth with the respiratory activity of $B$. stearothermophilus. NADH and ascorbate$N, N, N^{\prime}, N$ '-tetramethyl-p-phenylenediamine (ascorbateTMPD) were chosen as substrates because they allowed to study the full respiratory system (NADH) or only the final oxidase segment (ascorbate-TMPD). 


\section{Materials and methods}

\subsection{Cultures}

The strain of B. stearothermophilus and conditions of its maintenance have been described previously (Jurado et al., 1987). B. stearothermophilus was grown at $65^{\circ} \mathrm{C}$ in a complex medium (L-broth) to which tamoxifen from a concentrated ethanolic solution was added to obtain concentrations ranging from 2.5 to $7.5 \mu \mathrm{M}$, before inoculation. The bacterial growth in media containing tamoxifen is performed in silanized Erlenmeyer flasks, since this drug strongly binds to glass material (Custódio et al., 1991). Growth was measured by turbidimetry at $610 \mathrm{~nm}$ in a Spectronic 201 instrument.

\subsection{Measurement of oxygen consumption rate}

Oxygen consumption was measured with protoplasts prepared from cells from cultures harvested in the middle of the exponential growth phase, by incubation with lysozyme, essentially as described by Wisdom and Welker (1973). The protein content of the protoplasts was determined by the Biuret method, calibrated with bovine serum albumin (Gornal et al., 1949). The respiratory activity of the protoplasts was monitored polarographically with a Clark oxygen electrode (Estabrook, 1967) connected to a recorder, in a thermostated water-jacketed closed chamber $(1 \mathrm{ml})$, with magnetic stirring, at $37^{\circ} \mathrm{C}$. TAM as dimethyl sulfoxide

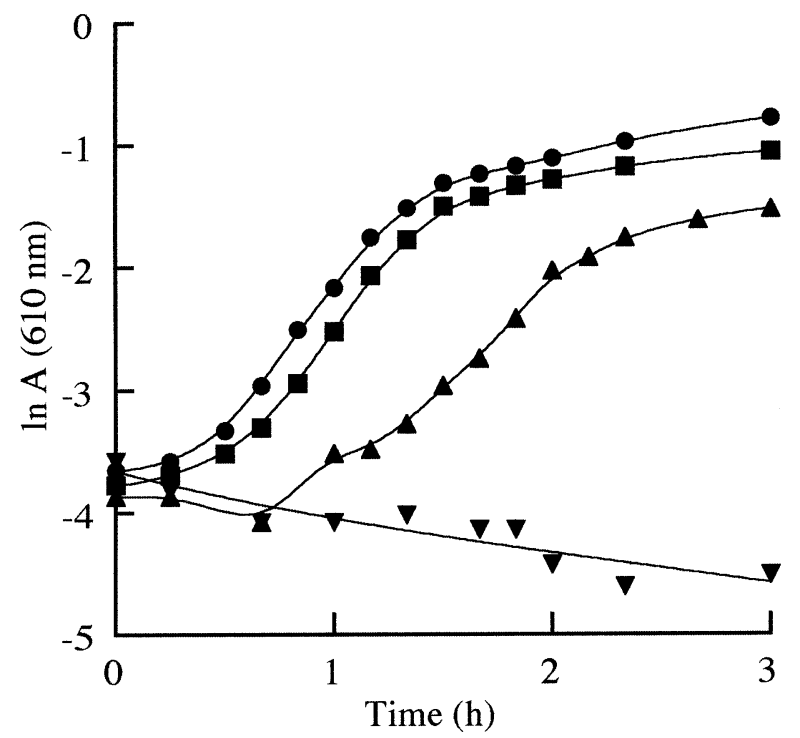

Fig. 1. Effects of tamoxifen on the growth of Bacillus stearothermophilus at $65^{\circ} \mathrm{C}$ (optimal growth temperature). Cells were grown in the control medium (O) and in media containing tamoxifen at final concentrations of $2.5 \mu \mathrm{M}(\boldsymbol{\square}), 5 \mu \mathrm{M}(\boldsymbol{\Delta})$ and $7.5 \mu \mathrm{M}(\boldsymbol{\nabla})$. Growth was measured as optical density at $610 \mathrm{~nm}$. The results shown are typical of three separate experiments. solution was added to the reaction medium $(40 \mathrm{~mm}$ HEPES-Tris, $10 \mathrm{~mm} \mathrm{MgCl}_{2}$; $\mathrm{pH}$ 7.5) with the protoplasts $(0.5 \mathrm{mg}$ protein), and each preparation was incubated for $4 \mathrm{~min}$, before the addition of the substrate. The effect of TAM on oxygen consumption of protoplasts was examined using $10 \mathrm{~mm}$ NADH or $10 \mathrm{~mm}$ ascorbate plus $600 \mu \mathrm{M}$ TMPD as substrates. These substrates allowed the study of the NADH or only the final oxidase segment (ascorbate-TMPD). Controls were prepared without the drug but with the same volume of dimethyl sulfoxide, the drug solvent.
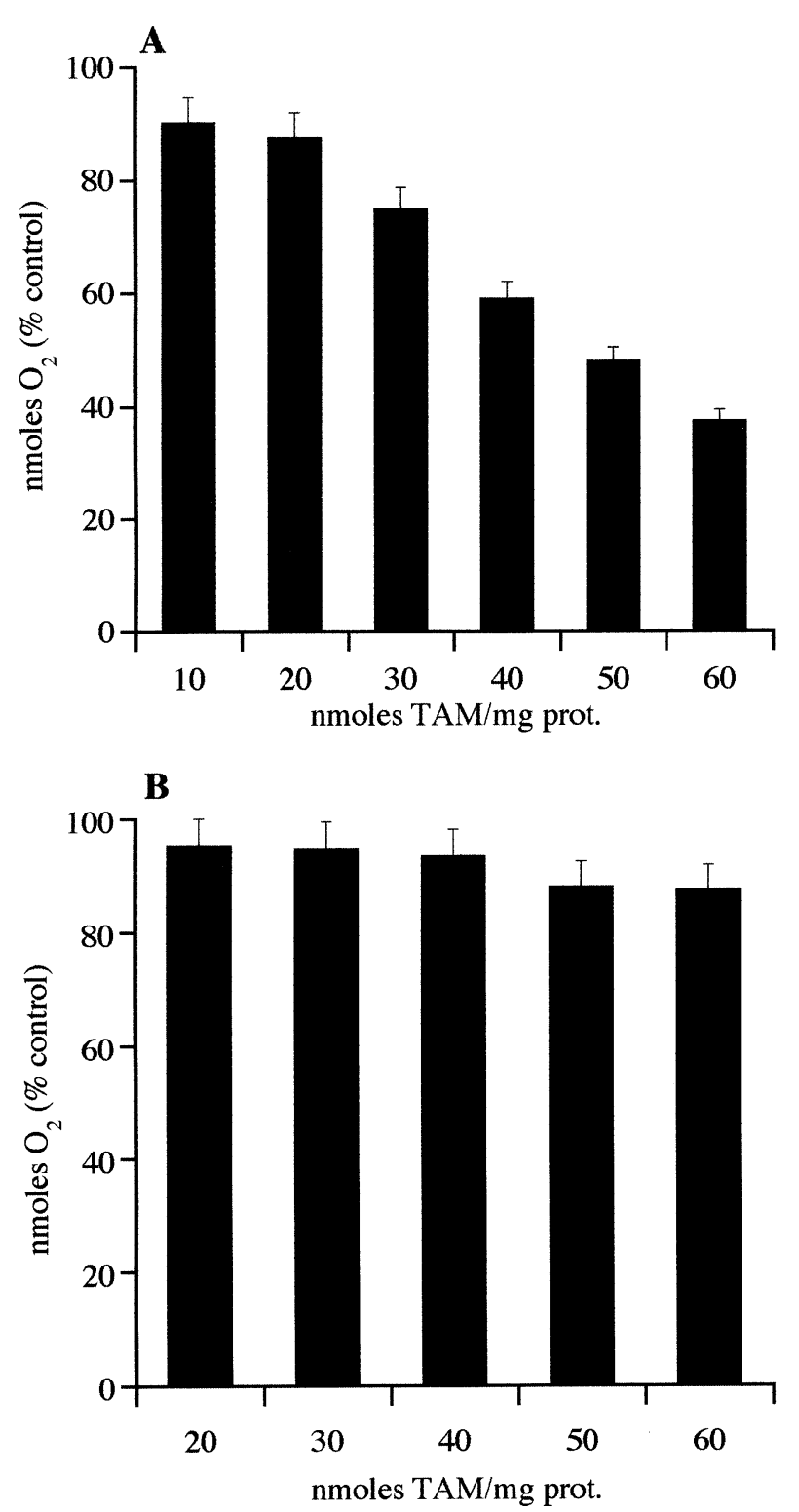

Fig. 2. Effects of tamoxifen on the oxygen consumption rate of $B$. stearothermophilus protoplasts using NADH (A) or ascorbate-TMPD (B) as substrate. The bars display the levels of the respiratory rate compared to the control (protoplasts incubated without TAM). The values are the means \pm S.D. from three to four different experiments. 


\section{Results}

\subsection{Effects of TAM on the growth of B. stearothermophilus}

Growth of B. stearothermophilus in a complex medium (L-broth) at $65^{\circ} \mathrm{C}$ (within the optimal temperature range) was inhibited by the addition of TAM as a function of concentration (ranging from 2.5 to $7.5 \mu \mathrm{M}$ ). Growth with increasing drug concentrations was characterized by progressively longer lag periods, lower specific growth rates and lower bacterial yields. The addition of $7.5 \mu \mathrm{M}$ TAM completely inhibited growth (Fig. 1).

\subsection{Effects of TAM on the $\mathrm{O}_{2}$ consumption rate of $B$. stearothermophilus protoplasts}

The effects of TAM on the oxygen consumption rate of protoplasts are shown in Fig. 2. When NADH was used as substrate (Fig. 2A), significant inhibition of the oxygen consumption rate was induced by TAM. The inhibition was concentration dependent in the range of 10 to $60 \mathrm{nmol} \mathrm{TAM} / \mathrm{mg}$ of protein. The oxygen consumption supported by ascorbate-TMPD was less sensitive to inhibition by TAM. At the maximum concentration assayed, levels of inhibition of 62.5 and $12.6 \%$ were obtained when NADH or ascorbate-TMPD were used as substrates, respectively. As expected, the $\mathrm{O}_{2}$ consumption supported by NADH and ascorbateTMPD was completely inhibited by the addition of $\mathrm{KCN}$ (1 $\mathrm{mm})$, as a consequence of full inhibition of cytochrome oxidase.

\section{Discussion}

The effects of TAM on growth and the respiratory activity of $B$. stearothermophilus are tentatively correlated. TAM inhibits bacterial growth as a function of concentration and exerted a significant inhibitory effect on $\mathrm{O}_{2}$ consumption of protoplasts. The inhibitory effects of TAM on respiratory activity depend on the substrate used, with the oxygen consumption supported by NADH being more sensitive to the inhibition by TAM than ascorbate-TMPD support respiratory. These preliminary data suggest that the interaction of TAM with the respiratory components occurs at a level preceding the cytochrome oxidase segment.

The toxic effects of TAM on bacterial bioenergetics along with bacterial growth impairment and membrane structure perturbations (Luxo et al., 1996, 1998) may underlie the mechanism of TAM antiproliferative action and of its antitumoral activity.

\section{References}

Clarke, R., van den Berg, H.W., Murphy, R.F., 1990. Reduction of the membrane-fluidity of human breast cancer cells by tamoxifen and 17ß-estradiol. Journal of the National Cancer Institute 82, 1702-1705.

Custódio, J.B.A., Almeida, L.M., Madeira, V.M.C., 1993. The anticancer drug tamoxifen induces changes in the physical properties of model and native membranes. Biochimica et Biophysica Acta 1150, 123-129.

Estabrook, R.W., 1967. Mitochondrial respiratory control and the polarographic measurement of $\mathrm{ADP} / \mathrm{o}$ ratios. Methods in Enzymology 10, 41-47.

Gornal, A.G., Bardawill, C., David, M.M., 1949. Dtermination of serum proteins by means of the biuret method. Journal of Biological Chemistry $177,751-756$

Jurado, A.S., Santana, A.C., Costa, M.C., Madeira, V.M.C., 1987. Influence of divalent cations on the growth and morphology of Bacillus stearothermophilus. Journal of General Microbiology 133, 507-513.

Kon, O.L., 1989. Estrogens, antiestrogens and cell proliferation. BioEssays 10, 210-214.

Luxo, C., Jurado, A.S., Custódio, J.B.A., Madeira, V.M.C., 1996. Use of Bacillus stearothermophilus as a model to study tamoxifen-membrane interactions. Toxicology in Vitro 10, 463-471.

Luxo, C., Jurado, A.S., Madeira, V.M.C., 1998. Lipid composition changes induced by tamoxifen in a bacterial model system. Biochimica et Biophysica Acta 1369, 71-84.

van den Koedijk, C.D.M.A., Blankenstein, M.A., Thijssen, J.H.H., 1994. Speculation on the mechanism of action of triphenylethylene antioestrogens. Biochemical Pharmacology 47, 1927-1937.

Wisdom, C., Welker, N.E., 1973. Membranes of Bacillus stearothermophilus: factors affecting protoplast stability and thermostability of alkaline phosphatase and reduced nicotinamide adenine dinucleotide oxidase. Journal of Bacteriology 144, 1336-1345. 